\title{
Review of: "The effect of video-instructed versus audio-instructed dispatcher-assisted cardiopulmonary resuscitation on patient outcomes following out of hospital cardiac arrest in Seoul"
}

\author{
Hidetada Fukushima
}

Potential competing interests: The author(s) declared that no potential competing interests exist.

Thank you for the opportunity to review this manuscript.

First, there is a huge selection bias for Video instruction group.

As authors described in the Methods section, the Video instruction was assigned if: 1) there are two or more rescuers, 2) the second rescuers can use video function of the smartphones.

Although authors adjusted these variables in the analysis, this criteria can assign video instructions more often for the CA cases which multiple rescuers are available, the rescuers are likely to be young or at public places where generally the survival rates are higher.

These factors are in favor of the better survival.

I agree that video instruction can help rescuers perform more correct and effective CPR.

However, this study shows the additional effect of video instruction to the standard DACPR process when it is available, but not the effect of video instruction itself.

Second, I wonder how long generally it takes to start the Video instruction. Until the dispatcher starts the video instruction, there should be no difference in key time intervals between both groups. Since DACPR process is time-sensitive, it is quite important to show the key time intervals in both study groups. 